\title{
On the approximation hardness of geodetic set and its variants
}

\author{
Tom Davot ${ }^{1}$, Lucas Isenmann ${ }^{2}$, and Jocelyn Thiebaut ${ }^{3}$ \\ 1 LIRMM, Univ Montpellier, CNRS, Montpellier, France davot@lirmm.fr \\ 2 Aix-Marseille Université, France lucas .isenmann@laposte.net \\ 3 INSA Centre Val de Loire, Univ. Orléans, LIFO EA 4022, Orléans, France \\ jocelyn.thiebaut@univ-orleans.fr
}

\begin{abstract}
Given a graph, a geodetic set (resp. edge geodetic set) is a subset of its vertices such that every vertex (resp. edge) of the graph is on a shortest path between two vertices of the subset. A strong geodetic set is a subset $\mathrm{S}$ of vertices and a choice of a shortest path for every pair of vertices of S such that every vertex is on one of these shortest paths. The geodetic number (resp. edge geodetic number) of a graph is the minimum size of a geodetic set (resp. edge geodetic set) and the strong geodetic number is the minimum size of a strong geodetic set. We first prove that, given a subset of vertices, it is $\mathcal{N} \mathcal{P}$-hard to determine whether it is a strong geodesic set. Therefore, it seems natural to study the problem of maximizing the number of covered vertices by a choice of a shortest path for every pair of a provided subset of vertices. We provide a tight 2approximation algorithm to solve this problem. Then, we show that there is no $781 / 780$ polynomial-time approximation algorithm for edge geodetic number and strong geodetic number on subcubic bipartite graphs with arbitrarily high girth. We also prove that geodetic number and edge geodetic number are both LOG- $\mathcal{A} \mathcal{P} \mathcal{X}$-hard, even on subcubic bipartite graphs with arbitrarily high girth. Finally, we disprove a conjecture of Iršič and Konvalinka by proving that the strong geodetic number can be computed in polynomial time in complete multipartite graphs.
\end{abstract}

\section{Introduction}

Geodetic number and edge geodetic number. A geodesic between two vertices of a graph $G$ is a path of minimum length between $x$ and $y$. The geodetic number of $G$ is the minimum size of a subset $X$ of the vertices such that, for every vertex $v$, there exists a geodesic between two vertices of $X$ containing $v$. The geodetic number of a graph has been introduced by Harary et al in [13], where the authors showed that deciding whether a graph has a geodetic number less than an integer $k$ is $\mathcal{N} \mathcal{P}$-complete. The complexity of this problem has also been investigated in several classes of graphs, such as bipartite graphs [11] and chordal graphs [10]

Preprint Version

The final authentificated version is available online at https://doi.org/10.1007/

978-3-030-89543-3_7 
where it remains $\mathcal{N} \mathcal{P}$-complete. Recently, Chakraborty et al. proved that finding the geodetic number of a graph is $\mathcal{N} \mathcal{P}$-hard on planar graphs with maximum degree six and line graphs [6]. They also proved in [7] that, unless $\mathcal{P}=\mathcal{N} \mathcal{P}$, there is no polynomial time $o(\log n)$-approximation algorithm for computing the geodetic number of a graph, even on graphs that have a universal vertex and where $n$ stands for the number of vertices in the input graph.

The edge version of the geodetic number has been introduced independently in [3] and in [22]. A subset $X$ of the vertices is an edge geodetic set if, for every edge $e$, there is a geodesic between two vertices of $X$ containing $e$. The edge geodetic number of $G$ is the size of the smallest geodetic set of $G$. Note that, given a graph $G$, the geodetic number of $G$ is smaller than its edge geodetic number. This edge version is also known to be $\mathcal{N} \mathcal{P}$-hard [3]. This problem has been studied on several classes of graphs, such as Cartesian products [1,21] and fuzzy graphs [20]. From a structural point of view, Santhakumaran and Ullas Chandran characterized graphs with a prescribed edge geodetic number [23]. For more results and motivations about geodetic sets, see [5].

Strong geodetic number and strong edge geodetic number. A subset of vertices $X$ is a strong geodetic set if there exists a function $\tilde{I}$ that associates a unique geodesic to each pair of vertices of $X$ and such that every vertex $v$ is contained in a geodesic $\tilde{I}(a, b)$, where $\{a, b\} \subseteq X$. In the following, we call such a function a geodesic assignation for $X$. The strong geodetic number of a graph $G$ is the size of the smallest strong geodetic set. The strong geodetic number has been introduced recently by Arokiaraj et al. [2]. In their original paper, the authors motivate this variation by social network applications. Furthermore, they also prove that this problem is $\mathcal{N} \mathcal{P}$-complete. Note that it remains $\mathcal{N} \mathcal{P}$-hard even when restricted to bipartite graphs [15]. This problem has been studied on complete Apollonian networks [2], grids and cylinders [16], and on Cartesian product of graphs [12], on complete bipartite graphs [14], on complete multipartite graphs [15] and on outerplanar graphs [18]. Connections to the diameter of the graph were studied in [14] and to the isometric path problem [2].

Finally, the edge version of the strong geodetic problem, where we want to cover every edge of the graph, has been introduced by Manuel et al. [17] and were proved $\mathcal{N} \mathcal{P}$-complete. Zmazek recently studied the values of the edge strong geodetic number on grids [25].

Our results. The results of our paper are divided in four sections. In Section 3, we propose a variant of the strong geodetic problem where, given a subset $S$ of vertices, the question is to determine whether $S$ is a strong geodesic set of the graph. Using a reduction from Monotone BALANCED 3-SAT-(4), we prove that this problem is $\mathcal{N} \mathcal{P}$-hard. Then, we consider in Section 4 the problem of maximizing the number of covered vertices by a choice of a geodesic for each pair of a provided subset of vertices, and provide a tight 2-approximation algorithm to solve it. In Section 5, we reduce the geodetic problems from Set Cover. We first give it in the general case, and then we adapt the previous construction on bipartite graphs with arbitrarily high girth. Using the previous reductions, we show in Section 6 that there is no approximation of EdGe GeOdetic Number 
with an approximation factor better than $781 / 780$. We also prove that geodetic number and edge geodetic number are both LOG- $\mathcal{A} \mathcal{P} \mathcal{X}$-hard, even on subcubic bipartite graphs with arbitrarily high girth. Finally, in section 7, we give a polynomial time algorithm which computes the Strong GeOdetic Number of complete multipartite graphs, disproving the conjecture of [15] which states that Strong Geodetic Number is $\mathcal{N} \mathcal{P}$-hard on complete multipartite graphs.

Due to space constraints, the proofs have been omitted. However, a full version can be found in https://hal-lirmm.ccsd.cnrs.fr/lirmm-03328636.

\section{Notations}

We first introduce some notations and formally define the problems. Given a set $X$, we denote by $\mathcal{P}_{2}(X)$ the set of its pairs. Given two sets $X$ and $Y$, we denote by $X \sqcup Y$ the union $X \cup Y$ when $X$ and $Y$ are disjoint. Let $G$ be a graph, we denote by $V(G)$ its set of vertices and by $E(G)$ its set of edges. We denote by $D_{1}(G)$ the set of vertices of degree one in $G$. Let $X$ be a subset of vertices of $G$ and $x$ be a vertex, we say that $x$ is selected by $X$ if $x \in X$ and that $x$ is covered by $X$ if $x$ is contained in a geodesic between two vertices of $X$ (or simply selected or covered if there is no ambiguity on $X$ ). Likewise, let $u v$ be an edge, we say that $u v$ is covered by $X$ (or simply covered) if $u v$ is contained in a geodesic between two vertices of $X$.

To describe a path between two vertices $u$ and $v$, we introduce the operator $\sim$ as follows. Let $p_{1}, p_{2}, \ldots, p_{k}$ be some subgraphs such that $u \in p_{1}, v \in p_{k}$ and for each $i<k$, there exists a unique vertex $x_{i} \in p_{i} \cap p_{i+1}$. The path described by $p_{1} \sim p_{2} \sim \cdots \sim p_{k}$ corresponds to $\left(u, \ldots, x_{1}, \ldots, x_{i}, \ldots, x_{k-1}, \ldots, v\right)$ (following successively the paths $p_{1}, p_{2}, \ldots, p_{k}$ ). Let $g$ be a path that contains the vertices $u$ and $v$. We denote by $g[u, v]$ the subpath of $g$ with extremities $u$ and $v$. Furthermore, we denote by $V(g)$ the vertices of $g$. Similarly, given a geodesic assignation $\tilde{I}$ for a set of vertices $X$, we denote by $V(\tilde{I})$ the vertices covered by the geodesics of $\tilde{I}$.

We now introduce the problems studied in this work.

(Strong) (Edge) Geodetic Number

Input: a simple graph $G$ and an integer $k$.

Question: is there a (strong) (edge) geodetic set $X \subseteq V$ of size $k$ ?

The following already known property will be fundamental in the proofs of our reductions as it helps to force some vertices to be part of a (strong) (edge) geodetic set.

Property 1. If $G$ is a graph and $X$ is a solution of any geodesic problem, then we have $D_{1}(G) \subseteq X$.

\section{Hardness to find a geodesic assignation}

In the proof that computing the strong geodesic number is $\mathcal{N} \mathcal{P}$-complete, the geodesic assignation is rather trivial [2]. In this section, we show that determining 
if a set of vertices is a strong geodetic set (i.e. computing a geodesic assignation) is in itself $\mathcal{N} \mathcal{P}$-complete. To do so, we reduce from a special case of 3 -SAT called Monotone BALANCEd 3-SAT-(4). In this variant, the boolean formula is composed of monotone clauses, that is, clauses that contains only positive literals or only negative literals. Monotone BALANCED 3-SAT-(4) is defined as follows.

Monotone Balanced 3-SAT-(4)

Input: a monotone 3-SAT formula $\varphi$ where each variable occurs exactly two times positively and two times negatively.

Question: is $\varphi$ satisfiable?

Darman and Döcker showed that this problem is $\mathcal{N} \mathcal{P}$-complete [8]. We introduce the following construction.

Construction 1. Let $\varphi$ be a Monotone Balanced 3-SAT-(4) formula, we construct the following graph $G$ :

- For each clause $C_{j}$, introduce a vertex $q_{j}$.

- For each variable $x_{i}$, introduce two edges $v_{i}^{0} v_{i}^{1}$ and $u_{i}^{0} u_{i}^{1}$. Furthermore, let $C_{j}$ and $C_{j^{\prime}}$, with $j<j^{\prime}$ be the two clauses where $x_{i}$ occurs with the same polarity (i.e. it appears positively in both clauses, or negatively in both), construct a path $\left(v_{i}^{1}, q_{j}, q_{j^{\prime}}, u_{i}^{1}\right)$.

- For each pair of vertices $v_{i}^{1}$ and $u_{i^{\prime}}^{1}$ with $i \neq i^{\prime}$, introduce a vertex $t_{i, i^{\prime}}$ and construct the path $\left(v_{i}^{1}, t_{i, i^{\prime}}, u_{i^{\prime}}^{1}\right)$.

- Finally, construct two vertices $k_{v}$ and $k_{u}$, and for each variable $x_{i}$, introduce the edges $v_{i}^{1} k_{v}$ and $u_{i}^{1} k_{u}$.



For each variable $x_{i}$, let $C_{j}$ and $C_{j^{\prime}}$ (resp. $C_{k}$ and $C_{k^{\prime}}$ ) with $j<j^{\prime}$ be the clauses where $x_{i}$ occurs positively (resp. negatively). We denote by $p_{i}$ the path $\left(v_{i}^{0}, v_{i}^{1}, q_{j}, q_{j^{\prime}}, u_{i}^{1}, u_{i}^{0}\right)$, by $\bar{p}_{i}$ the path $\left(v_{i}^{0}, v_{i}^{1}, q_{k}, q_{k^{\prime}}, u_{i}^{1}, u_{i}^{0}\right)$ and by $p_{i, i^{\prime}}$ the path $\left(v_{i}^{0}, v_{i}^{1}, t_{i, i^{\prime}}, u_{i^{\prime}}^{1}, u_{i^{\prime}}^{0}\right)$, for any $i \neq i^{\prime}$. An example of a graph produced by Construction 1 is depicted in Figure 1.

Lemma 1. Let $\varphi$ be a Monotone Balanced 3-SAT-(4) formula and $G$ its graph resulting from Construction 1. Let $\tilde{I}$ be a geodesic assignation for $D_{1}(G)$. 
It is possible to construct a geodesic assignation $\tilde{I}^{\prime}$ for $D_{1}(G)$ such that $\left|V\left(\tilde{I}^{\prime}\right)\right| \leq$ $|V(\tilde{I})|$, and:

(1) for any $i \neq i^{\prime}$, the geodesic between $v_{i}^{0}$ and $u_{i^{\prime}}^{0}$ in $\tilde{I}^{\prime}$ is $p_{i, i^{\prime}}$, and

(2) for any $i$, the geodesic between $v_{i}^{0}$ and $u_{i}^{0}$ in $\tilde{I}^{\prime}$ is either $p_{i}$ or $\bar{p}_{i}$.

Proof. First, note that every geodesic with extremity $v_{i}^{0}$ (resp. $u_{i}^{0}$ ) covers the vertex $v_{i}^{1}$ (resp. $u_{i}^{1}$ ). Second, the geodesic between two vertices $u_{i}^{0}$ and $u_{i^{\prime}}^{0}$ is $\left(u_{i}^{0}, u_{i}^{1}, k_{u}, u_{i^{\prime}}^{1}, u_{i^{\prime}}^{0}\right)$. Similarly, the geodesic between two vertices $v_{i}^{0}$ and $v_{i^{\prime}}^{0}$ is $\left(v_{i}^{0}, v_{i}^{1}, k_{v}, v_{i^{\prime}}^{1}, v_{i^{\prime}}^{0}\right)$. Hence, it remains to cover the $q_{j}$ vertices and the $t_{i, i^{\prime}}$ vertices. Let $V_{1}=\left\{v_{i}^{1} \mid x_{i} \in \varphi\right\}$ and $U_{1}=\left\{u_{i}^{1} \mid x_{i} \in \varphi\right\}$. Since there is no edge between $V_{1}$ and $U_{1}$, the distance between any vertex of $V_{1}$ and any vertex of $U_{1}$ is at least two. From that, we can deduce that no geodesic $g$ between two vertices $v_{i}^{0}$ and $u_{i^{\prime}}^{0}$ contains the vertex $k_{v}$ or the vertex $k_{u}$ since the length of $g$ would be at least six, and $p_{i, i^{\prime}}$ or $p_{i}$ (if $i=i^{\prime}$ ) are shorter paths. Let us now prove the two items of the statement.

(1) Since the distance between $V_{1}$ and $U_{1}$ is at least two, the path $p_{i, i^{\prime}}$ is a geodesic between $v_{i}^{0}$ and $u_{i^{\prime}}^{0}$. Suppose that there exists another geodesic $g$ between $v_{i}^{0}$ and $u_{i^{\prime}}^{0}$. We have $g=\left(v_{i}^{0}, v_{i}^{1}, q_{j}, u_{i^{\prime}}^{1}, u_{i^{\prime}}^{0}\right)$, where the clause $C_{j}$ contains $x_{i}$ and $x_{i^{\prime}}$ in $\varphi$. Suppose, without loss of generality that $C_{j}$ contains positive literals. Toward a contradiction, suppose that $t_{i, i^{\prime}}$ is covered by $\tilde{I}$ and let $g^{\prime}$ be the geodesic that covers $t_{i, i^{\prime}}$. Since $g^{\prime}$ cannot have the same extremities as $g, g^{\prime}$ contains either the vertex $k_{v}$ or the vertex $k_{u}$, contradicting $g^{\prime}$ being a geodesic. Hence, the vertex $t_{i, i^{\prime}}$ is not covered by $\tilde{I}$, then we can replace $g$ by $p_{i, i^{\prime}}$ in it to obtain a solution as thought.

(2) Let $g$ be a geodesic between $v_{i}^{0}$ and $u_{i}^{0}$. Since, $g$ does not contain $k_{v}$, then $v_{i}^{1}$ is adjacent to a vertex $q_{j}$ in $g$. Likewise, $u_{i}^{1}$ is adjacent to a vertex $q_{j^{\prime}}$ in $g$. By contradiction, suppose that $x_{i}$ appears positively in $C_{j}$ and negatively in $C_{j^{\prime}}$. Then, since the edge $q_{j} q_{j^{\prime}}$ exists, by construction, there is a path $p_{i^{\prime}}$ or $\bar{p}_{i^{\prime}}$ containing $q_{j}$ and $q_{j^{\prime}}$. But then, the variable $x_{i^{\prime}}$ appears either positively in $C_{j}$ and $C_{j^{\prime}}$ or negatively in $C_{j}$ and $C_{j}^{\prime}$. In any case, we reach a contradiction since either $C_{j}$ or $C_{j^{\prime}}$ is not monotone. Hence, $x_{i}$ appears only positively in $C_{j}$ and $C_{j^{\prime}}$ or only negatively in $C_{j}$ and $C_{j^{\prime}}$, and then $g$ corresponds to either $p_{i}$ or $\bar{p}_{i}$.

Theorem 1. It is $\mathcal{N P}$-hard to determine if a set of vertices $V^{\prime}$ is a strong geodetic set even if, for every strong geodetic set $V_{\text {strong }}$, we have $V^{\prime} \subseteq V_{\text {strong }}$.

Proof. Let $\varphi$ be a Monotone Balanced 3-SAT-(4) formula and $G$ its graph resulting from Construction 1 . We show that $\varphi$ is satisfiable if and only if $D_{1}(G)$ is a strong geodetic set.

- Let $\beta$ be a satisfying assignment of $\varphi$, we construct a geodesic assignation $\tilde{I}$ for $D_{1}(G)$ as follows. For each $i \neq i^{\prime}$, we set $\tilde{I}\left(v_{i}^{0}, u_{i}^{0}\right)=p_{i, i^{\prime}}, \tilde{I}\left(v_{i^{\prime}}^{0}, u_{i}^{0}\right)=p_{i^{\prime}, i}$, $\tilde{I}\left(v_{i}^{0}, v_{i^{\prime}}^{0}\right)=\left(v_{i}^{0}, k_{v}, v_{i^{\prime}}^{0}\right)$, and $\tilde{I}\left(u_{i}^{0}, u_{i^{\prime}}^{0}\right)=\left(u_{i}^{0}, k_{u}, u_{i^{\prime}}^{0}\right)$. Hence, every vertex is covered except the $q_{j}$ vertices. Now, for each variable $x_{i}$, if $x_{i}$ is assigned to true, we set $\tilde{I}\left(v_{i}^{0}, u_{i}^{0}\right)=p_{i}$ and we set $\tilde{I}\left(v_{i}^{0}, u_{i}^{0}\right)=\bar{p}_{i}$, otherwise. 
Suppose, there is a vertex $q_{j}$ that is not covered by $\tilde{I}$. Let $x_{i}, x_{k}$ and $x_{\ell}$ be the three variables that occur in $C_{j}$ and suppose that $C_{j}$ contains positive literals. We have $i m g(\tilde{I}) \cap\left\{p_{i}, p_{k}, p_{\ell}\right\}=\emptyset$, and then $\beta\left(x_{i}\right)=\beta\left(x_{k}\right)=\beta\left(x_{\ell}\right)=$ false, contradicting $\beta$ being a satisfying assignment for $\varphi$. Hence, every vertex of $G$ is covered and $D_{1}(G)$ is a strong geodetic set of $G$.

- Let $\tilde{I}$ be a geodesic assignation for $D_{1}(G)$ that covers every vertex of $G$, and such that $\tilde{I}$ respects properties of Lemma 1 . We construct a satisfying assignment $\beta$ for $\varphi$ as follows. For each variable $x_{i}$, if $p_{i} \in i m g(\tilde{I})$, we set $\beta\left(x_{i}\right)=$ true and $\beta\left(x_{i}\right)=$ false, otherwise.

Suppose there is a clause $C_{j}$ that is not satisfied by $\beta$ and suppose by symmetry that $C_{j}$ contains positive literals. Let $x_{i}, x_{k}$ and $x_{\ell}$ be the three literals of $C_{j}$. We have $\operatorname{img}(\tilde{I}) \cap\left\{p_{i}, p_{k}, p_{\ell}\right\}=\emptyset$ and then $q_{j}$ is not covered by $\tilde{I}$ which is a contradiction. Hence, $\beta$ is a satisfying assignment for $\varphi$.

Finally, by Property 1 , the set $D_{1}(G)$ belongs to any strong geodetic set.

From this theorem, we can derive a result about the residue variant of Strong Geodetic Number. The residue variant of an optimisation problem has been defined recently in [24] and consists of, given a partial solution $P$ for an instance $I$, finding an optimal partial solution $R$ such that $P \cup R$ is a solution for $I$. The complexity class $\mathcal{R} \mathcal{A} \mathcal{P} \mathcal{X}$ contains the residue variant optimisation problems such that the score of the residue can be approximated by a constant.

\section{Corollary 1. Strong Geodetic Number $\notin \mathcal{R} \mathcal{A P \mathcal { X }}$}

Proof. Let $\varphi$ be a Monotone BAlanced 3-SAT-(4) formula and $G$ be its graph resulting from Construction 1. Then, given a partial solution $P=D_{1}(G)$, $G$ has a residue solution $R=\emptyset$ if and only if $\varphi$ is satisfiable. Hence, the residual variant of Strong Geodetic Number cannot be approximated to any constant factor unless $\mathcal{P}=\mathcal{N} \mathcal{P}$.

\section{Approximation}

Since it is hard to determine if a subset of vertices is a strong geodetic set, a natural question that arises is to find, given a subset of vertices, a geodetic assignation that maximizes the number of covered vertices. We call this problem Max Geodesic Assignation. By Theorem 1, this problem is also $\mathcal{N} \mathcal{P}$-hard and we show that this problem belongs to $\mathcal{A P \mathcal { X }}$, i.e. approximable within a constant ratio. In this part, we show that this problem is 2-approximable using a simple greedy algorithm, defined in Algorithm 1.

Theorem 2. Algorithm 1 computes in polynomial time a solution for MAX Geodesic Assignation with an approximation ratio of 2 and this ratio is tight.

Proof. Let $\tilde{I}_{a p p}$ be the geodesic assignation computed by Algorithm 1 and let $\tilde{I}_{o p t}$ be an optimal geodesic assignation. We show that there exists an application $f: V\left(\tilde{I}_{\text {opt }}\right) \rightarrow V\left(\tilde{I}_{\text {app }}\right)$ such that for each $u \in V\left(\tilde{I}_{\text {app }}\right),\left|f^{-1}(u)\right| \leq 2$. 


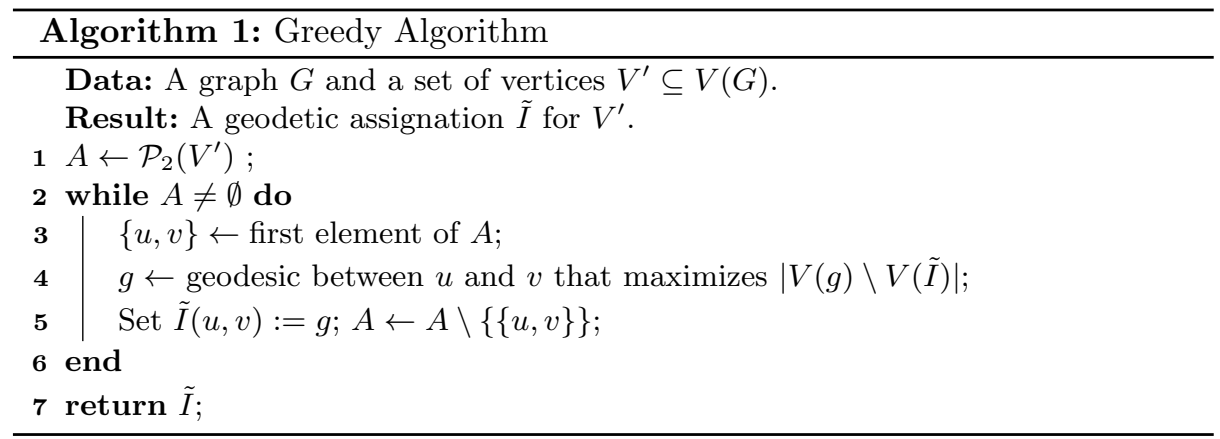

First, for each vertex $v \in V\left(\tilde{I}_{o p t}\right) \cap V\left(\tilde{I}_{a p p}\right)$, we set $f(v)=v$. Further, let $g_{a p p}^{i}$ the geodesic chosen by the greedy algorithm at step $i$ and let $g_{\text {opt }}^{i}$ be the geodesic with the same extremities in $\tilde{I}_{o p t}$. For each $i$, let $V_{o p t}^{i}=V\left(g_{o p t}^{i}\right) \backslash \bigcup_{j<i} V\left(g_{o p t}^{j}\right)$ and $V_{a p p}^{i}=V\left(g_{a p p}^{i}\right) \backslash \bigcup_{j<i} V\left(g_{a p p}^{j}\right)$ (i.e. the set of vertices newly covered by $\left.g_{a p p}^{i}\right)$. We have $\left|V_{o p t}^{i} \backslash V\left(\tilde{I}_{a p p}\right)\right| \leq\left|V_{a p p}^{i}\right|$ since otherwise, the greedy algorithm would have chosen $g_{\text {opt }}^{i}$ at step $i$. Thus, there exists an injective function $f^{\prime}$ : $V_{o p t}^{i} \backslash V\left(\tilde{I}_{a p p}\right) \rightarrow V_{a p p}^{i}$ and, for each vertex $v \in V_{o p t}^{i} \backslash V\left(\tilde{I}_{a p p}\right)$, we set $f(v)=f^{\prime}(v)$. Since each vertex $u \in V\left(\tilde{I}_{\text {app }}\right)$ belongs to a unique $V_{a p p}^{i}$, we have $\left|f^{-1}(u)\right| \leq 2$. Moreover, since each vertex $v \in V\left(\tilde{I}_{o p t}\right)$ belongs either to $V\left(\tilde{I}_{o p t}\right) \cap V\left(\tilde{I}_{a p p}\right)$ or to a set $V_{o p t}^{i} \backslash V\left(\tilde{I}_{a p p}\right)$, we defined a function $f$ as thought. It follows that $\left|V\left(\tilde{I}_{o p t}\right)\right| \leq 2 \cdot\left|V\left(\tilde{I}_{\text {app }}\right)\right|$, proving the approximation ratio. Furthermore, the ratio is tight, as shown by Figure 2.



Fig. 2. Tightness of the approximation ratio of Algorithm 1. Consider $a, c$ and $e$ as selected (in black in the graph). The optimal solution consists in taking the geodesics $(a, b, e),(c, d, e)$ and $(a, c, d)$ which cover the non-selected vertices $b$ and $d$. The greedy algorithm can start by taking the geodesic $(a, d, e)$ between $a$ and $e$. Then the algorithm will choose $(c, d, e)$ and $(a, c, d)$ for the last two pairs. This leads to a set of geodesics which only covers $d$.

\section{Reduction from Set Cover}

In this part, we prove preliminary results that will be used in the next section. More specifically, we reduce the geodetic problems from the classic $\mathcal{N} \mathcal{P}$-complete problem SET COVER described as follows. 

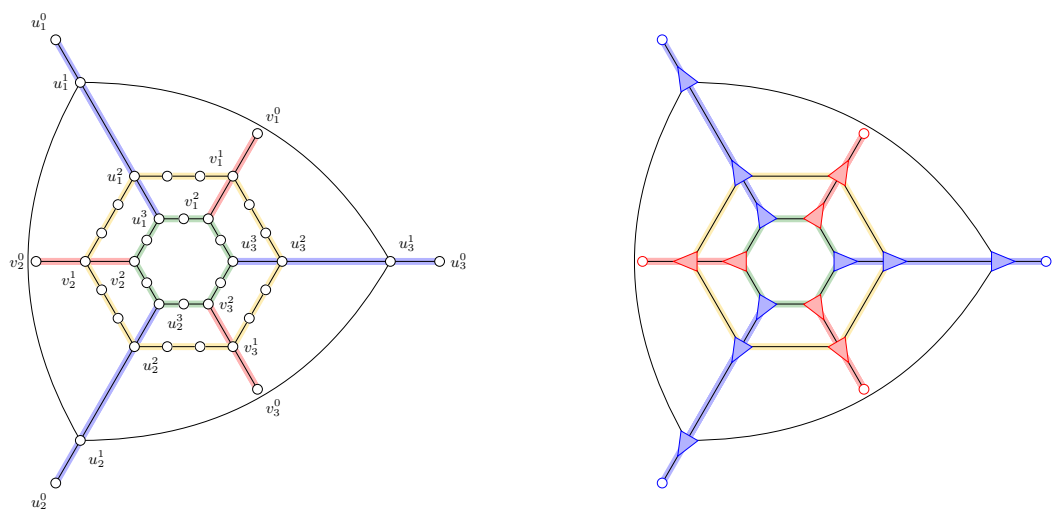

Fig. 3. Example of a graph produced by Construction 2 (left) and Construction 4 (right) on the collection containing $S_{1}=\left\{E_{1}, E_{3}\right\}, S_{2}=\left\{E_{1}, E_{2}\right\}$ and $S_{3}=\left\{E_{2}, E_{3}\right\}$. Element paths, set paths, cut paths and long paths are coloured in blue, red, yellow and green, respectively. In the right graph, black edges, green edges and yellow edges represent paths of length $h, 2 h$ and $3 h$, respectively

Set Cover (SC)

Input: A collection $C=\left\{S_{1}, \ldots, S_{m^{\prime}}\right\}$ of finite sets over the universe $U=\left\{E_{1}, \ldots, E_{n^{\prime}}\right\}$.

Question: Find a minimum $C^{\prime} \subseteq C$ such that every element of $U$ is contained in a set of $C^{\prime}$.

For the strong versions, we use a version of SET Cover, denoted $\left(k, k^{\prime}\right)$-SET Cover, where the size of the intersection between two sets is at most $k$ and the set sizes are bounded by $k^{\prime}$. Notice that since VerTex Cover is a particular case of $\left(1, k^{\prime}\right)$-Set Cover, then $\left(k, k^{\prime}\right)$-Set Cover is $\mathcal{N} \mathcal{P}$-complete.

In the following, we first show how this reduction works in the general case and then, we adapt it in subcubic bipartite graphs with arbitrary high girth.

\subsection{On general case}

Construction 2. Let $(C, U)$ be an instance of SET Cover. We construct a graph $G$ as follows:

- For each set $S_{i}$, create a 3-path $s p_{i}=\left(v_{i}^{0}, v_{i}^{1}, v_{i}^{2}\right)$.

- For each element $E_{j}$, create a 4-path $e p_{j}=\left(u_{j}^{0}, u_{j}^{1}, u_{j}^{2}, u_{j}^{3}\right)$. We denote the edge $u_{j}^{2} u_{j}^{3}$ as $e_{j}$.

- For each set $S_{i}$ and each element $E_{j} \in S_{i}$, introduce a 3-path $c p_{j}^{i}$ between $v_{i}^{1}$ and $u_{j}^{2}$ ) and a 2-path $l p_{j}^{i}$ between $v_{i}^{2}$ and $u_{j}^{3}$.

- For each pair of elements $E_{j}$ and $E_{j^{\prime}}$, introduce the edge $t_{j, j^{\prime}}=u_{j}^{1} u_{j^{\prime}}^{1}$.

The paths $e p_{j}, s p_{i}, c p_{j}^{i}$ and $l p_{k}^{i}$ are called element paths, set paths, cut paths and long paths, respectively. An example of a graph produced by Construction 2 is depicted in Figure 3. 
Clearly, the construction can be carried in polynomial time. In order to show that Construction 2 constitutes a reduction, we introduce the following lemmas.

Lemma 2. Let $(C, U)$ be an instance of SeT Cover (resp. $\left(1, k^{\prime}\right)$-SET Cover) and let $G$ be its graph resulting from Construction 2. The set $D_{1}(G)$ covers (resp. strongly covers) every edge of $G^{\prime}$ except the edges in $\left\{e_{j} \mid E_{j} \in U\right\}$.

Proof. We start by describing the geodesics between each pair of vertices of $D_{1}(G)$ and how we can make the assignation them when there is some choice.

(a) For any pair of elements $E_{j}$ and $E_{j^{\prime}}$ of $U$, the distance between $u_{j}^{0}$ and $u_{j^{\prime}}^{0}$ is 3 and the unique geodesic between these two vertices is described by $e p_{j} \sim t_{j, j^{\prime}} \sim e p_{j^{\prime}}$.

(b) For any set $S_{i}$ of $C$ and any element $E_{j} \in S_{i}$, the distance between $u_{j}^{0}$ and $v_{i}^{0}$ is 6 and the unique geodesic between these two vertices is described by $e p_{j} \sim c p_{j}^{i} \sim s p_{i}$.

(c) For any set $S_{i}$ of $C$ and any element $E_{j} \notin S_{i}$, the distance between $u_{j}^{0}$ and $v_{i}^{0}$ is 7 and the geodesics are described by $e p_{j} \sim t_{j, j^{\prime}} \sim e p_{j^{\prime}} \sim c p_{j^{\prime}}^{i} \sim s p_{i}$, for any element $E_{j} \in S_{i}$. Since these geodesics contain only edges that are covered by the cases (a) or (b), we can assign any geodesic for this case.

(d) For any pair of sets $S_{i}$ and $S_{i^{\prime}}$ of $C$ such that $S_{i} \cap S_{i^{\prime}} \neq \emptyset$, the distance between $v_{i}^{0}$ and $v_{i^{\prime}}^{0}$ is 8 and the geodesics between these two vertices are described by $s p_{i} \sim c p_{j}^{i} \sim c p_{j}^{i^{\prime}} \sim s p_{i^{\prime}}$ and $s p_{i} \sim l p_{j}^{i} \sim l p_{j}^{i^{\prime}} \sim s p_{i^{\prime}}$, for each $E_{j} \in S_{i} \cap S_{i^{\prime}}$. Since, the edges of $c p_{j}^{i}$ and $c p_{j}^{i^{\prime}}$ are already covers by the case (b), it is better to assign a geodesic $s p_{i} \sim l p_{j}^{i} \sim l p_{j}^{i^{\prime}} \sim s p_{i^{\prime}}$, note that if $\left|S_{i} \cap S_{i^{\prime}}\right|=1$, then there only one such geodesic.

(e) For any pair of sets $S_{i}$ and $S_{i^{\prime}}$ of $C$ such that $S_{i} \cap S_{i^{\prime}}=\emptyset$, the distance between $v_{i}^{0}$ and $v_{i^{\prime}}^{0}$ is 11 and the geodesics between these two vertices are described by $s p_{i} \sim c p_{j}^{i} \sim e p_{j} \sim t_{j, j^{\prime}} \sim e p_{j^{\prime}} \sim c p_{j^{\prime}}^{i^{\prime}} \sim s p_{i^{\prime}}$, for any $E_{j} \in S_{i}$ and $E_{j^{\prime}} \in S_{i^{\prime}}$.

Remark that the $e_{j}$ edges are not covered by any geodesic between the vertices of $D_{1}(G)$. We conclude by showing that every other edges are covered by $D_{1}(G)$, even if we fix a unique geodesic in the case where the intersection between two sets is at most one.

Let $e$ be an edge in $E(G) \backslash\left\{e_{j} \mid E_{j} U\right\}$. If $e$ belongs to a set path $s p_{i}$ or a long path $l p_{i}$, then $e$ is covered by the geodesic between $v_{i}^{0}$ and $v_{j}^{0}$. If $e$ belongs to an element path $e p_{j}$ or a cut path $c p_{j}^{i}$, then $e$ is covered by the geodesic between $v_{i}^{0}$ and $u_{i, j}^{0}$. Finally, if $e$ is a $t_{j, j^{\prime}}$ edge, then $e$ is covered by the geodesic between $u_{j}^{0}$ and $u_{j^{\prime}}^{0}$. Hence, every edge of $G$ is covered except the $e_{j}$ edges.

In the following, let $Y_{i}^{S} \subset V(G)$ denote the set containing $s p_{i} \backslash\left\{v_{i}^{0}\right\}$ and every long path $l p_{j}^{i}$ and cut path $c p_{j}^{i}$ incident to $c p_{i}$ minus vertices of every element path $e p_{j}$. Formally, $Y_{i}=\left(s p_{i} \backslash\left\{v_{i}^{0}\right\}\right) \cup\left\{\left(c p_{j}^{i} \cup l p_{j}^{i}\right) \backslash e p_{j} \mid \forall E_{j} \in S_{i}\right\}$. For each element $E_{j} \in U$, we also denote $Y_{j}^{E}=\left\{Y_{i} \mid E_{j} \in S_{i}\right\} \cup e p_{j} \backslash\left\{u_{j}^{0}\right\}$. 
Lemma 3. Let $(C, U)$ be an instance of SET Cover and let $G$ be its graph resulting from Construction 2. For each element $E_{j}$, every geodesic containing the edge $e_{j}$ has an extremity in $Y_{j}^{E}$.

Proof. Toward a contradiction, we suppose that there exists an edge $e_{j}$ such that there is a geodesic $g$ with extremities $x \notin Y_{j}^{E}$ and $y \notin Y_{j}^{E}$ that contains $e_{j}$. For simplicity, we denote the subpath $g\left[x, u_{j}^{3}\right]$ as $g_{x}$ (we suppose that $e_{j} \notin g_{x}$ ). By hypothesis, $g_{x}$ contains a long path $l p_{j}^{i}$ such that $E_{j} \in S_{i}$. Since $g_{x}$ can not have an extremity in $Y_{i}^{S}, g_{x}$ contains a vertex $x^{\prime}$ in some element path $e p_{k}$, such that $E_{k} \in S_{i}$. The subpath $g\left[x^{\prime}, u_{j}^{2}\right]$ can be described either by $c p_{k}^{i} \sim s p_{i} \sim c p_{j}^{i} \sim e p_{j}$ or by $l p_{k}^{i} \sim s p_{i} \sim l p_{j}^{i} \sim e p_{j}$. Let $g^{\prime}$ be the path between $x^{\prime}$ and $u_{j}^{2}$ described by $e p_{k} \sim t_{k, j} \sim e p_{j}$. By construction, we have $\left|g^{\prime}\right|<\left|g\left[x^{\prime}, u_{j}^{2}\right]\right|$. Thus replacing $g\left[x^{\prime}, u_{j}^{2}\right]$ by $g^{\prime}$ in $g$ constructs a path between $x$ and $y$ that is shorter than $g$, contradicting that $g$ is a geodesic. Hence, $X$ contains at least one vertex in $Y_{j}^{E}$.

In order to easily produce a set cover in $G$ from a (strong) edge geodetic set $X$ of $G^{\prime}$, we need $X$ to respect a certain property. Hence, we use the following lemma.

Lemma 4. Let $(C, U)$ be an instance of Set Cover (resp. $\left(1, k^{\prime}\right)$-Set Cover) and $G$ its graph resulting from Construction 2. Let $X \subseteq V(G)$ be an edge geodetic set (resp. strong edge geodetic set) of $G$. It is possible to construct an edge geodetic set (resp. a strong edge geodetic set) $X^{\prime}$ of $G$ such that $\left|X^{\prime}\right| \leq|X|$ and

$$
X^{\prime} \subseteq\left\{v_{i}^{2} \mid S_{i} \in C\right\} \cup D_{1}(G)
$$

Proof. First by Property 1, $X$ contains any vertices of $D_{1}(G)$. Therefore, by Lemma 2, every edge is (strongly) covered by $D_{1}(G)$ except the $e_{j}$ edges. Note that selecting a vertex $v_{i}^{2}$ ensures to cover every $e_{j}$ edges (with $E_{j} \in S_{i}$ ) since the unique geodesic between $v_{i}^{1}$ and $u_{j}^{0}$ is described by $l p_{j}^{i} \sim e p_{j}$.

We show how to transform $X$ so that it respects lemma's property. For each edge $e_{j}$, by Lemma 3, there is a vertex $x$ in the intersection $X \cap Y_{j}^{E}$. If $x \in Y_{i}^{S}$ for some $S_{i}$ such that $E_{j} \in S_{i}$, we replace $x$ in $X$ by $v_{i}^{2}$. Since $x$ belongs to any set $Y_{k}^{E}$ such that $S_{k} \in E_{i}$, every edge previously covered by $x$ is still covered. If $x \in e p_{j}$ then it is only used to cover $e_{j}$. Thus, we can replace $x$ arbitrarily by any vertex $v_{i}^{2}$ such that $E_{j} \in S_{i}$. Finally, there exists a vertex that does not belong to $\left\{v_{i}^{2} \mid S_{i} \in C\right\} \cup D_{1}(G)$, we can remove it from $X$. Hence, we obtain a solution as thought.

Lemma 5. Let $(C, U)$ be an instance of Set Cover (resp. $\left(1, k^{\prime}\right)$-SeT Cover) and $G$ its graph resulting from Construction 2. Then the instance $(C, U)$ contains a set cover of size $k$ if and only if $G$ contains an edge geodetic set (resp. strong edge geodetic set) of size $k+|C|+|U|$.

Proof. - Let $C^{\prime} \subseteq C$ be a set cover of size $k$ of $(C, U)$ and consider the (strong) edge geodetic set $X=D_{1}(G) \cup\left\{v_{i}^{2} \mid S_{i} \in C^{\prime}\right\}$. By Lemma 2, every edges 
in $E(G) \backslash\left\{e_{j} \mid E_{j} \in U\right\}$ are (strongly) covered. Let $E_{j}$ be an element of $U$, there is a set $S_{i} \in S^{\prime}$ such that $E_{j} \in S_{i}$. Thus, $v_{i}^{2} \in X$ and therefore the edge $e_{j}$ is covered by the unique geodesic between $v_{i}^{2}$ and $u_{i, j}^{0}$. Since every element of $U$ appears in $S^{\prime}$, every $e_{j}$ edges of $G$ are also (strongly) covered. Hence, we produce a (strong) edge geodetic set of size $k+|C|+|U|$.

- Let $X \subset V(G)$ be a (strong) edge geodetic set of size $k+|C|+|U|$ of $G$ that respects the property of Lemma 4 . Consider the set cover $C^{\prime}=\left\{S_{i} \mid v_{i}^{2} \in X\right\}$. Let $E_{j}$ be an element of $U$ and suppose that it does not belong to a set of $C^{\prime}$. In that case, no vertex $v_{i}^{2}$ such that $E_{j} \in S_{i}$ belongs to $X$. By Lemma 3 , the edge $e_{j}$ is not covered by $X$, contradicting $X$ being a (strong) edge geodetic set. Hence, every element of $U$ is contained in a set of $C^{\prime}$ and, we construct a set cover of size $k$.

\subsection{On subcubic bipartite graphs}

We now extend the previous result to subcubic and bipartite graphs. First, we show that the result holds in graph with maximum degree three. We introduce the following construction.

Construction 3. Given a graph $G$, a vertex $u \in V(G)$, a set of non-adjacent neighbours $N^{0}=\left\{v_{0}^{0}, \ldots, v_{k-1}^{0}\right\} \subseteq N(u)$ and an integer $h>\log k$, emplace $a$ $h$-pyramid $P y\left(h, u, N^{0}\right)$ consists of removing all edges between $u$ and $N^{0}$ and replacing them with the following subgraph. For each $0<i<h$, construct recursively the sets $N^{i}$ :

- create $t=\left\lceil\left|N^{i-1}\right| / 2\right\rceil$ vertices $v_{0}^{i}, \ldots, v_{t}^{i}$, and

- introduce the edges $v_{t^{\prime}}^{i} v_{2 t^{\prime}}^{i-1}$, and $v_{t^{\prime}}^{i} v_{2 t^{\prime}+1}^{i-1}$ (if $v_{2 t^{\prime}+1}^{i-1}$ exists) for each $t^{\prime}<t$.

Finally, introduce the edge $u v_{0}^{h-1}\left(N^{h-1}\right.$ consists of a single vertex since $h>$ $\log k)$.
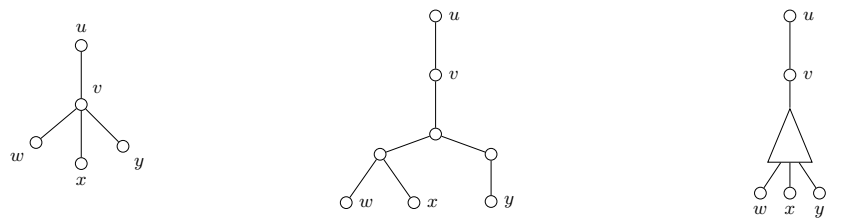

Fig. 4. Example of a 3-pyramid $P y(3, v,\{w, x, y\})$ produced by Construction 3. Left: $v$ and its neighbours in the original graph. Center: emplaced 3-pyramid. Right: Representation of the pyramid used in Figure 3.

Let $P y(h, v, N)$ be a $h$-pyramid. We can make the following observations.

- The maximum degree of $P y_{h}(h, v, N)$ is three.

- Let $n_{1}, n_{2} \in N$, the distance between $n_{1}$ and $n_{2}$ in $P y_{h}(h, v, N)$ is between 2 and $2 h$ and the distance between $v$ and $n_{1}$ or $n_{2}$ is $h$. 
We now use the previous structure to modify Construction 2 as follows.

Construction 4. Let $(C, U)$ be an instance of SET COVER and $G$ be its graph resulting from Construction 2. Let $h>\log \Delta(G)$ be an integer. We modify $G$ as follows:

- for each set $S_{i}$ and each integer $k \in\{1,2\}$, emplace a h-pyramid $P y\left(h, v_{i}^{k}, N\left(v_{i}^{k}\right) \backslash\left\{v_{i}^{k-1}\right\}\right)$,

- for each element $E_{j}$ and each integer $k \in\{1,2,3\}$, emplace a h-pyramid $P y\left(h, u_{j}^{k}, N\left(u_{j}^{k}\right) \backslash\left\{u_{j}^{k-1}\right\}\right)$, and

- replace each edge of $G$ that does not belong to a $h$-pyramid by a path of length $h$.

Note that the resulting graph has maximum degree three. Moreover, if $k$ is odd then the resulting graph is bipartite. Finally, by taking an arbitrary high value of $k$, the resulting graph has an arbitrary high girth. We use a similar vocabulary than for Construction 2: an element tree et ${ }_{j}$ is the tree induced by the vertices of $e p_{j}$ in the $h$-pyramids emplaced in it in the original graph. A set tree $s t_{i}$ is defined the same way. For each element $E_{j}$, the $h$-path that replaces the edge $e_{j}$ is denoted $p_{j}$. An example of a graph produced by Construction 4 is depicted in Figure 3. Since Construction 4 multiplies the length of every path of Construction 2 by $h$, we can adapt Lemmas 2 to 4 to it by replacing $e p_{j}$ by $e t_{j}$ and $s p_{i}$ by $s t_{i}$ in the geodesics descriptions.

Lemma 6. Let $(C, U)$ be an instance of SET Cover (resp. $\left(1, k^{\prime}\right)$-Set Cover) be a connected graph and $G$ its graph resulting from Construction 4 . The set $D_{1}(G)$ covers (resp. strongly cover) every edge and vertex of $G$ except edges and vertices in $\left\{p_{j} \mid E_{j} \in U\right\}$.

Proof. By construction, geodesics between vertices of $D_{1}(G)$ can be described the same way as for Lemma 2. The result follows.

In the following $Y_{i}^{S}$ and $Y_{j}^{E}$ are defined in the same way as for Construction 2 (by taking $s t_{i}$ instead of $s p_{i}$ and $e t_{j}$ instead of $e p_{j}$ ). Notice that the geodesic between a vertex $v^{1}$ and a vertex $u_{j}^{0}$ is described by $s t_{i} \sim l p_{j}^{i} \sim e t_{j}$ and contains the path $p_{j}$. Hence, using the same arguments as in Lemmas 3 and 4 , we can show the two following results.

Lemma 7. Let $(C, U)$ be an instance of SET COVER and $G$ be its graph resulting from Construction 4 . For each element $E_{j}$, every geodesic containing a vertex of $p_{j}$ has an extremity in $Y_{j}^{E}$.

Lemma 8. Let $(C, U)$ be an instance of SET Cover (resp. $\left(1, k^{\prime}\right)$-SET Cover) and $G$ be its graph resulting from Construction 4. Let $X \subseteq V(G)$ be a geodetic or an edge geodetic set of $G^{\prime}$ (resp. strong geodetic or a strong edge geodetic set). It is possible to construct a geodetic or an edge geodetic set (resp. strong geodetic or a strong edge geodetic set) $X^{\prime}$ of $G$ such that $\left|X^{\prime}\right| \leq|X|$ and

$$
X^{\prime} \subseteq\left\{v_{i}^{2} \mid S_{i} \in C\right\} \cup D_{1}(G) .
$$


Using the same idea as for Lemma 5, we can now show that Construction 4 constitutes a reduction: if a path $p_{j}$ is (strongly) covered, then there is a vertex $v_{i}^{2}$, such that $E_{j} \in S_{i}$, is selected. Thus, given a solution for a geodetic problem $X$ the set $\left\{S_{i} \mid v_{i}^{2} \in X\right\}$ is a set cover of $G$. Hence, we obtain the following result.

Lemma 9. Let $(C, U)$ be an instance of Set Cover (resp. $\left(1, k^{\prime}\right)$-Set Cover) and $G$ its graph resulting from Construction 2. Then the instance $(C, U)$ contains a set cover of size $k$ if and only if $G$ contains an edge geodetic set and a geodetic set (resp. strong edge geodetic set and a strong geodetic set) of size $k+|C|+|U|$.

\section{Non-approximability}

In this section, we use the results of the previous section to find hardness of approximation results for the geodetic problems.

\subsection{Strong geodetic set and strong edge geodetic set}

First, recall the definition of $L$-reduction between two hard problems $\Pi$ and $\Pi^{\prime}$ (with respective cost functions $v a l_{\Pi}$ and $v a l_{\Pi^{\prime}}$ ), as described by Papadimitriou and Yannakakis [19]. Let $O P T_{\Pi}(x)$ and $O P T_{\Pi^{\prime}}(x)$ be the optimal value of $v a l_{\Pi}$ and $v a l_{\Pi^{\prime}}$ on an instance $x$, respectively. An $L$-reduction consists of polynomialtime computable functions $f$ and $g$ such that, for each instance $x$ of $\Pi, f(x)$ is an instance of $\Pi^{\prime}$ and for each feasible solution $y^{\prime}$ for $f(x), g\left(y^{\prime}\right)$ is a feasible solution for $x$. Moreover, there are constants $\alpha_{1}, \alpha_{2}>0$ such that:

1. $O P T_{\Pi^{\prime}}(f(x)) \leq \alpha_{1} \cdot O P T_{\Pi}(x)$ and

2. $\left|\operatorname{val}_{\Pi}\left(g\left(y^{\prime}\right)\right)-O P T_{\Pi}(x)\right| \leq \alpha_{2} \cdot\left|v a l_{\Pi^{\prime}}\left(y^{\prime}\right)-O P T_{\Pi^{\prime}}(f(x))\right|$.

Using Construction 4 , we obtain an $L$-reduction with $\alpha_{1}=\left(2 k^{\prime}+2\right)$ and $\alpha_{2}=1$.

Lemma 10. Let $\rho_{k^{\prime}}$ be the best possible polynomial time approximation factor of $\left(1, k^{\prime}\right)$-Minimum Set Cover. Then Strong Geodetic Number and Strong EdGe GeOdetic Number cannot be approximated with a factor better than

$$
1+\frac{\rho_{k^{\prime}}-1}{2 k^{\prime}+2},
$$

in subcubic bipartite graphs with arbitrary high girth.

Proof. Let $(C, U)$ be an instance of $\left(1, k^{\prime}\right)$-Minimum Set Cover and $G$ its graph resulting from Construction 4. Suppose there is a polynomial-time approximation algorithm for Strong Geodetic Number (resp. Strong Edge Geodetic NUMBER) in subcubic bipartite graphs with arbitrary high girth and let $X_{a p p}$ be a strong geodetic set (resp. strong edge geodetic set) computed by this algorithm in $G$. We suppose that $X_{a p p}$ respects the property of Lemma 8. Let $C_{a p p}^{\prime}=\left\{S_{i} \mid\right.$ $\left.v_{i}^{2} \in X_{a p p}\right\}$. Using the same argument as in Lemma 9, we can show that $C_{a p p}^{\prime}$ is a set cover of $(C, U)$ and $\left|X_{a p p}\right|=|C|+|U|+\left|C_{a p p}^{\prime}\right|$. Let $C_{o p t}^{\prime} \subset C$ be a set cover of $(C, U)$ and let $X_{\text {opt }}$ be a minimum strong geodetic set (resp. strong edge 
geodetic set) in $G$. Similarly, we can show that $\left|X_{\text {opt }}\right|=|C|+|U|+\left|C_{\text {opt }}^{\prime}\right|$. Hence, we have

$$
\left|X_{a p p}\right|-\left|X_{o p t}\right|=\left|C_{a p p}^{\prime}\right|-\left|C_{o p t}^{\prime}\right| .
$$

Moreover, since the intersection between two sets contains at least two elements and the size of each set is bounded by $k^{\prime}$, we deduce

$$
|U| \leq k^{\prime} \cdot\left|C_{o p t}^{\prime}\right| \text { and }|C| \leq\left(k^{\prime}+1\right) \cdot\left|C_{o p t}^{\prime}\right|
$$

which leads to

$$
\left|X_{o p t}\right| \leq\left(2 k^{\prime}+2\right) \cdot\left|C_{o p t}^{\prime}\right| .
$$

Thus we construct a $L$-reduction with $\alpha_{1}=\left(2 k^{\prime}+2\right)$ and $\alpha_{2}=1$. We conclude,

$$
\begin{aligned}
\left|X_{a p p}\right| & \stackrel{(1)}{=}\left|C_{a p p}^{\prime}\right|-\left|C_{o p t}^{\prime}\right|+\left|X_{o p t}\right| \\
& \geq\left(\rho_{k^{\prime}}-1\right) \cdot\left|C_{o p t}^{\prime}\right|+\left|X_{o p t}\right| \\
& \stackrel{(2)}{\geq}\left(1+\frac{\rho_{k^{\prime}}-1}{2 k^{\prime}+2}\right) \cdot\left|X_{o p t}\right| .
\end{aligned}
$$

Since Minimum Vertex Cover with bounded maximum degree $k^{\prime}$ is a particular case of $\left(1, k^{\prime}\right)$-Minimum Set Cover, we can pick the value of $k^{\prime}$ (and so the corresponding best-known value $\rho_{k^{\prime}}$ ) that maximize the previous inapproximation ratio. Thus, since Berman and Karpinski showed that Minimum VERTEX COVER cannot be approximated with a factor better than 79/78 in graphs with maximum degree four [4], we obtain the following result.

Corollary 2. Strong Geodetic Number and Strong Edge Geodetic NUMBER cannot be approximated with a factor better than 781/780 in subcubic bipartite graphs with arbitrary high girth.

\subsection{Geodetic set and edge geodetic set}

Now, we provide approximation lower bounds for Geodetic Number and Strong Geodetic Number. We apply the following modification to Construction 4 .

Construction 5. Let $(C, U)$ be an instance of Set Cover, $G$ be its graph produced by Construction 4 and $k>|V(G)|$ be an integer. We construct a graph $G^{\prime}$ as follows:

- create $k$ disjoint copies $\left\{G_{1}, \ldots, G_{k}\right\}$ of $G$,

- for each vertex $x$ of $D_{1}(G)$,

- create an edge $s_{x}^{0} s_{x}^{1}$,

- for each $G_{\ell} \in\left\{G_{1}, \ldots, G_{k}\right\}$ and for each vertex $x \in D_{1}\left(G_{\ell}\right)$, construct a k-path $p_{x}^{\ell}$ between $x$ and $s_{x}^{1}$, and

- emplace a k-pyramid $P y\left(k, s_{x}^{1}, N\left(s_{x}^{1}\right) \backslash\left\{s_{x}^{0}\right\}\right)$. 
Notice that the resulting graph has maximum degree three. For simplicity, we denote the $k$-pyramide $P y\left(k, s_{x}^{1}, N\left(s_{x}^{1}\right) \backslash\left\{s_{x}^{0}\right\}\right)$ as $P y[x]$.

Lemma 11. Let $(C, U)$ be an instance of SET Cover and let $G^{\prime}$ be its graphs resulting from Construction 5. The set $D_{1}\left(G^{\prime}\right)$ covers every edge and vertex of $G^{\prime}$ except edges and vertices in $\left\{p_{j} \mid E_{j} \in U, G_{\ell} \in\left\{G_{1}, \ldots, G_{k}\right\}, p_{j} \in G_{\ell}\right\}$.

Proof. Let $G$ be the graph of $(C, U)$ produced Construction 4. Let $x \in D_{1}(G)$ and $G_{\ell}$ in $\left\{G_{1}, \ldots, G_{k}\right\}$. The shortest path between $G_{\ell}$ and $s_{x}^{0}$ has length $2 k$ and walks through $p_{k}^{\ell}$ and $P y[x]$. Let $x_{1}$ and $x_{2}$ be two vertices of $D_{1}(G)$ and $x_{1}^{\prime}$ and $x_{2}^{\prime}$ be their corresponding vertices in $G_{\ell}$. Let $g_{\ell}$ be a geodesic between $x_{1}^{\prime}$ and $x_{2}^{\prime}$, note that $g_{\ell}$ is entirely contained in $G_{\ell}$ since every path leaving $G_{\ell}$ has length $k>\left|V\left(G_{\ell}\right)\right|$. Suppose that $g=s_{x_{1}}^{0} s_{x_{1}}^{1} \sim P y\left[x_{1}\right] \sim p_{x_{1}}^{\ell} \sim g_{\ell} \sim p_{x_{2}}^{\ell} \sim P y\left[x_{2}\right] \sim$ $s_{x_{2}}^{0} s_{x_{2}}^{1}$ is not a geodesic between $s_{x_{1}}^{0}$ and $s_{x_{2}}^{0}$. Then, let $g^{\prime}$ be a geodesic between $s_{x_{1}}^{0}$ and $s_{x_{2}}^{0}$. If $g^{\prime}$ contains two subgraph $G_{\ell}$ and $G_{\ell^{\prime}}$, then since the distance between $G_{\ell}$ and $G_{\ell^{\prime}}$ is $2 k$, the length of $g^{\prime}$ is at least $6 k$ and then $g$ is shorter than $g^{\prime}$. Thus, $g^{\prime}$ contains only one subgraph $G_{\ell}$ and then, it can be described by $s_{x_{1}}^{0} s_{x_{1}}^{1} \sim P y\left[x_{1}\right] \sim p_{x_{2}}^{\ell} \sim g_{\ell}^{\prime} \sim p_{x_{2}}^{\ell} \sim P y\left[x_{2}\right] \sim s_{x_{2}}^{0} s_{x_{2}}^{1}$, where $g_{\ell}^{\prime}$ is a path between $x_{1}^{\prime}$ and $x_{2}^{\prime}$ in $G_{\ell}$, but then it contradicts $g_{\ell}$ being a geodesic. Hence, $g$ is a geodesic between $s_{x_{1}}^{0}$ and $s_{x_{2}}^{0}$. From that, we can conclude that $D_{1}\left(G^{\prime}\right)$ covers exactly the same edges and vertices than $D_{1}(G)$ in each $G_{\ell}$. Hence, by Lemma 6 , every edge and vertex in any $G_{\ell}$ is covered, except edges and vertices in $\left\{p_{j} \mid E_{j} \in U\right\}$.

It remains to show that edges and vertices in every $P y[x]$ and $p_{x}^{\ell}$ are also covered. First, since for every $x \in D_{1}(G)$ and every $G_{\ell}$, there is a geodesic $g$ with extremity $s_{x}^{0}$ that walks through $G_{\ell}$, then $g$ contains $p_{x}^{\ell}$ and thus every edge and vertex of $p_{x}^{\ell}$ is covered. Moreover, since every $p_{x}^{\ell}$ is adjacent to a distinct leaf of $P y[x]$, the set of geodesic with extremity $s_{x}^{0}$ covers every edge and vertex of $P y[x]$. The result follows.

Lemma 12. Let $(C, U)$ be an instance of SET COVER and let $G^{\prime}$ be its graph resulting from Construction 5. Let $X \subseteq V\left(G^{\prime}\right)$ be a geodetic set or an edge geodetic set of $G^{\prime}$. It is possible to construct a geodetic set or an edge geodetic set $X^{\prime}$ of $G^{\prime}$ such that $\left|X^{\prime}\right| \leq|X|$ and

$$
X^{\prime} \subseteq\left\{v_{i}^{2} \mid S_{i} \in C, G_{\ell} \in\left\{G_{1}, \ldots, G_{k}\right\}, v_{i}^{2} \in V\left(G_{\ell}\right)\right\} \cup D_{1}\left(G^{\prime}\right) .
$$

Proof. Since for each $G_{\ell} \in\left\{G_{1}, \ldots, G_{k}\right\}$, the result of Lemma 7 holds in $G_{\ell}$, we can use the same technique as for Lemma 8 to obtain a set $X^{\prime}$ such that $X^{\prime} \cap V\left(G_{\ell}\right)=\left\{v_{i}^{2} \mid S_{i} \in C\right\}$. Then, the result follows.

SeT Cover is hard to approximate with a factor better than a logarithmic function [9]. Therefore, we can transfer the lower bounds of approximation of Set Cover to Geodetic Number and Edge Geodetic Number. This result is in addition to the one proved by Chakraborty et al. [7].

Theorem 3. Geodetic Number and Edge Geodetic Number are LOG$\mathcal{A P} \mathcal{X}$-hard, even in bipartite subcubic graphs with arbitrary high girth. 
Proof. Let $(C, U)$ be an instance Set Cover graph and $G^{\prime}$ be its graph produced by Construction 5. Suppose that we have a polynomial-time approximation algorithm $A$ to compute a geodetic set or an edge geodetic set of a graph. We denote by $X$ the geodetic set obtained by $A$ in $G^{\prime}$ and we suppose that $X$ respects Lemma 12 property. We can suppose that $\left|X \cap V\left(G_{\ell}\right)\right|$ has the same value for any $G_{\ell} \in\left\{G_{1}, \ldots, G_{k}\right\}$, since otherwise it suffixes to transpose the solution with the minimum $\left|X \cap V\left(G_{\ell}\right)\right|$ value to the every other $G_{\ell}$. Let $C^{\prime}=\left\{S_{i} \mid v_{i}^{2} \in X \cap V\left(G_{1}\right)\right\}$. Using the same argument as in the proof of Lemma 5 , we can show that $C$ is a set cover of $(C, U)$, and that $|X|=k\left|C^{\prime}\right|+|C|+|U|$.

Let $X_{o p t}$ be a minimum geodetic set or a minimum edge geodetic set of $G^{\prime}$ and $C_{o p t}^{\prime}$ be a minimum set cover of $(C, U)$. Similarly, we can show that $\left|X_{\text {opt }}\right|=k\left|C_{\text {opt }}^{\prime}\right|+|U|+|C|$. We have:

$$
\frac{|X|}{\left|X_{\text {opt }}\right|}=\frac{k\left|C^{\prime}\right|+|U|+|C|}{k\left|C_{o p t}^{\prime}\right|+|U|+|C|}
$$

As $k$ tends to $+\infty$ we deduce that $\frac{|X|}{\left|X_{o p t}\right|}$ converges to $\left|C^{\prime}\right| /\left|C_{o p t}^{\prime}\right|$. Since SET Cover cannot be approximated with a factor better than a logarithmic function, we deduce that $A$ cannot have an approximation factor better than a logarithmic function.

\section{Strong Geodetic Number on complete multipartite graphs}

First, remark that geodesics of complete multipartite graphs are easy to determine: for any pair of vertices which are not in the same part, the edge between them is the unique shortest path between them. For a pair of vertices which are in the same part, the shortest paths between them are all the paths of length two between them with all the vertices not in this part as middle vertices.

In this section, we develop a polynomial algorithm which computes the strong geodetic number of a complete multipartite graph. The algorithm is based on dynamic programming where we not only look after a minimum strong geodetic set of vertices covering all the graph, but we look after all sets of vertices maximizing the number of pairs not used to cover other vertices among sets with some fixed parameters.

Let $K_{n_{1}, \ldots, n_{r}}$ denotes a complete multipartite graph whose parts are noted $X_{1}, \ldots, X_{r}$ such that $\left|X_{i}\right|=n_{i}$ for every $i \in\{1, \ldots, r\}$. We denote $N_{i}=\sum_{j=1}^{i} n_{j}$ for every $i \in\{1, \ldots, r\}$ and $K_{n_{1}, \ldots, n_{i}}$ by $G_{i}$.

Definition 1. A selection of $K_{n_{1}, \ldots, n_{r}}$ is a set of selected vertices $S$ in which we pick a set of pairs of non-adjacent vertices $C$ to cover some non-selected vertices. Formally, a selection is a triplet $(S, C, f)$, where

$-S \subseteq V$,

$-C \subseteq \bigcup_{j=1}^{r} \mathcal{P}_{2}\left(S \cap X_{j}\right)$ and, 
- $f: C \rightarrow V \backslash S$ is an injective map such that $\forall c \in C \cap \mathcal{P}_{2}\left(S \cap X_{i}\right), f(c) \notin X_{i}$ (i.e. two vertices of $X_{i}$ can not cover another vertex of $X_{i}$ ).

Given a selection $s(S, C, f)$, we denote by

- $s(S, C, f)=|S|$, the number of selected vertices,

- $r(S, C, f)=|V \backslash(S \sqcup f(C))|=n-s(S, C, f)-|C|$, the number of vertices that are neither selected nor covered, and

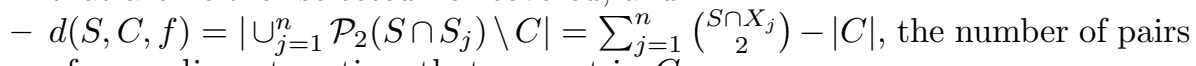
of non-adjacent vertices that are not in $C$.

We say that a selection $(S, C, f)$ is nice if it maximizes the number $d(S, C, f)$ among all selection with the same size $s(S, C, f)$ and the same number $r(S, C, f)$. In our dynamic programming approach, we construct every nice selections in every subgraph $G_{i}$, successively. To construct a selection in $G_{i}$ from a selection of $G_{i-1}$, we use the following lemma.

Lemma 13. Let $\left(S^{\prime}, C^{\prime}, f^{\prime}\right)$ a selection of $G_{i-1}$. Let three integers $k, u$ and $q$ such that $0 \leq k \leq n_{i}, 0 \leq u \leq \min \left(n_{i}-k, d\left(S^{\prime}, C^{\prime}, f^{\prime}\right)\right)$ and $0 \leq q \leq$ $\min \left(\left(\begin{array}{c}k \\ 2\end{array}\right), r\left(S^{\prime}, C^{\prime}, f^{\prime}\right)\right)$. There exists a selection $(S, C, f)$ of $G_{i}$ such that

$$
\left\{\begin{array}{l}
s(S, C, f)=s\left(S^{\prime}, C^{\prime}, f^{\prime}\right)+k \\
r(S, C, f)=r\left(S^{\prime}, C^{\prime}, f^{\prime}\right)+n_{i}-k-q-u \\
d(S, C, f)=d\left(S^{\prime}, C^{\prime}, f^{\prime}\right)+\left(\begin{array}{c}
k \\
2
\end{array}\right)-q-u .
\end{array}\right.
$$

In other words, we select $k$ vertices in $X_{i}$, cover $u$ vertices in $X_{i}$ and cover $q$ vertices in $G_{i-1}$.

Proof. Let $T$ be any subset of $X_{i}$ of size $k$. Let $Q$ be any subset of $\mathcal{P}_{2}(T)$ of size $q$. Let $U$ be any subset of $\bigcup_{j=1}^{i-1} \mathcal{P}_{2}\left(S^{\prime} \cap X_{j}\right) \backslash C^{\prime}$ of size $u$. Let $Q^{\prime}$ be any subset of $\bigcup_{j=1}^{i-1} X_{j} \backslash\left(S^{\prime} \cup f^{\prime}\left(C^{\prime}\right)\right)$ of size $q$. Let $U^{\prime}$ be any subset of $X_{i} \backslash T$ of size $u$.

We define

$$
\begin{aligned}
& S=S^{\prime} \cup T \\
& C=C^{\prime} \cup Q \cup U \\
& f: C \rightarrow V \backslash S
\end{aligned}
$$

where the function $f$ is defined as follows. On $C^{\prime}, f_{\mid C^{\prime}}=f^{\prime}$. As $U$ (resp. $Q$ ) and $U^{\prime}$ (resp. $Q^{\prime}$ ) are of the same size, we can define $f_{\mid U}$ (resp. $f_{\mid Q}$ ) as any bijection between $U$ and $U^{\prime}$ (resp. $Q$ and $Q^{\prime}$ ).

Let us show that $(S, C, f)$ is a selection of $G_{i}$. As $S^{\prime} \subseteq \bigcup_{j=1}^{i-1} X_{j}$ and $T \subseteq X_{i}$, we deduce that $S \subseteq \bigcup_{j=1}^{i} X_{j}$. As $C^{\prime} \subseteq \bigcup_{j=1}^{i-1} \mathcal{P}_{2}\left(S \cap X_{j}\right), Q \subseteq \mathcal{P}_{2}\left(S \cap X_{i}\right)$ and $U \subseteq \bigcup_{j=1}^{i-1} \mathcal{P}_{2}\left(S \cap X_{j}\right)$, we deduce that $C \subseteq \bigcup_{j=1}^{i} \mathcal{P}_{2}\left(S \cap X_{j}\right)$

By definition of $f, f_{\mid C^{\prime}}, f_{\mid Q}$ and $f_{\mid U}$ are injective. Let $x, x^{\prime} \in C$ such that $f(x)=f\left(x^{\prime}\right)$. As $f(U) \subseteq X_{i}$ and $f(Q)$ and $f\left(C^{\prime}\right) \subseteq \bigcup_{j=1}^{i-1} X_{j}$, we deduce that we can suppose that $x \in Q$ and $x^{\prime} \in C^{\prime}$. As $f(x) \in Q^{\prime}$, we have $f(x) \notin f^{\prime}\left(C^{\prime}\right)$. It contradicts the fact that $f(x)=f\left(x^{\prime}\right) \in f^{\prime}\left(C^{\prime}\right)$ because $x^{\prime} \in C^{\prime}$. We conclude that $x=x^{\prime}$ and that $f$ is injective. 




Fig. 5. Description of the creation of selection $(S, C, f)$ of $G_{i}$ from the selection $\left(S^{\prime}, C^{\prime}, f^{\prime}\right)$ of $G_{i-1}$. An arrow between two set indicates that pairs of vertices of the source set are used to cover vertices of the target set.

Let $a \in C \cap \mathcal{P}_{2}\left(S \cap X_{i}\right)=Q$. Then $f(a) \in Q^{\prime} \subseteq \bigcup_{j=1}^{i-1} X_{j}$. Thus, $f(a) \notin X_{i}$. Let $a \in C \cap \mathcal{P}_{2}\left(S \cap X_{j}\right)=C^{\prime} \cup U$ for any $j<i$. If $a \in C^{\prime}$, then $f(a)=f^{\prime}(a) \notin X_{j}$ because $\left(S^{\prime}, C^{\prime}, f^{\prime}\right)$ is a selection of $G_{i-1}$. Otherwise, $a \in U$ and $f(a) \in X_{i}$ and thus $f(a) \notin X_{j}$.

We conclude that $(S, C, f)$ is a selection of $G_{i}$.

As $S=S^{\prime} \sqcup T$, we deduce that $s(S, C, f)=s\left(S^{\prime}, C^{\prime}, f^{\prime}\right)+|T|=s\left(S^{\prime}, C^{\prime}, f^{\prime}\right)+$ $k$. As $C=C^{\prime} \sqcup Q \sqcup U$, we deduce that $r(S, C, f)=N_{i}-|S|-|C|=n_{i}+N_{i-1}-$ $\left|S^{\prime}\right|-k-\left|C^{\prime}\right|-|Q|-|U|=n_{i}-k-q-u+r\left(S^{\prime}, C^{\prime}, f^{\prime}\right)$. Furthermore

$$
\begin{aligned}
d(S, C, f) & =\sum_{j=1}^{i}\left(\begin{array}{c}
S \cap X_{j} \\
2
\end{array}\right)-|C| \\
& =\sum_{j=1}^{i-1}\left(\begin{array}{c}
S^{\prime} \cap X_{j} \\
2
\end{array}\right)+\left(\begin{array}{c}
|T| \\
2
\end{array}\right)-\left|C^{\prime}\right|-q-u \\
& =d\left(S^{\prime}, C^{\prime}, f^{\prime}\right)+\left(\begin{array}{c}
k \\
2
\end{array}\right)-q-u
\end{aligned}
$$

Since the number of selections can be exponential, we only keep nice selections. In order to do that, we adapt the result of Lemma 13 as follows.

Lemma 14. Let $(S, C, f)$ be a nice selection of $G_{i}$, then there exists a nice selection $\left(S^{\prime}, C^{\prime}, f^{\prime}\right)$ of $G_{i-1}$ and numbers $k, u, q$ such that $0 \leq k \leq n_{i}, 0 \leq q \leq$ $\min \left(\left(\begin{array}{l}k \\ 2\end{array}\right), r\left(S^{\prime}, C^{\prime}, f^{\prime}\right)\right)$ and $0 \leq u \leq \min \left(n_{i}-k, d\left(S^{\prime}, C^{\prime}, f^{\prime}\right)\right)$ and

$$
\left\{\begin{array}{l}
s(S, C, f)=s\left(S^{\prime}, C^{\prime}, f^{\prime}\right)+k \\
r(S, C, f)=r\left(S^{\prime}, C^{\prime}, f^{\prime}\right)+n_{i}-u-q-k \\
d(S, C, f)=d^{\prime}\left(S^{\prime}, C^{\prime}, f^{\prime}\right)+\left(\begin{array}{c}
k \\
2
\end{array}\right)-q-u
\end{array}\right.
$$

Proof. We define $S^{\prime}=S \bigcap G_{i-1}, C^{\prime}=C \bigcap G_{i-1} \backslash f^{-1}\left(X_{i}\right)$ and $f^{\prime}=f_{\mid C^{\prime}}$. Let us prove that $\left(S^{\prime}, C^{\prime}, f^{\prime}\right)$ is a nice selection of $G_{i-1}$ satisfying the announced equalities. 
Let us show that $\forall c \in C^{\prime} \cap \mathcal{P}_{2}\left(S \cap X_{i}\right), f^{\prime}(c) \notin X_{i}$. Let $c \in C^{\prime} \cap \mathcal{P}_{2}\left(S \cap X_{i}\right)$, suppose by contradiction that $f^{\prime}(c) \in X_{i}$. As $f^{\prime}(c)=f(c)$, we deduce that $c \in f^{-1}\left(X_{i}\right)$. This contradicts the definition of $C^{\prime}$. Thus, $f^{\prime}(c) \notin X_{i}$. As $f$ is injective, $f^{\prime}$ is also injective and, we deduce that $\left(S^{\prime}, C^{\prime}, f^{\prime}\right)$ is a selection of $G_{i-1}$.

We define $k=\left|S \cap X_{i}\right|, q=\left|C \cap \mathcal{P}_{2}\left(S \cap X_{i}\right)\right|$ and $u=\left|f^{-1}\left(X_{i}\right)\right|$. As $S=S^{\prime} \sqcup$ $\left(S \cap X_{i}\right)$, we have $s(S)=s\left(S^{\prime}\right)+k$. As we have $C=C^{\prime} \sqcup f^{-1}\left(X_{i}\right) \sqcup\left(C \cap \mathcal{P}_{2}\left(S \cap X_{i}\right)\right.$, thus $|C|=\left|C^{\prime}\right|+u+q$. We deduce that $r(S)=N_{i}-|S|-|C|=n_{i}+N_{i-1}-$ $s\left(S^{\prime}, C^{\prime}, f^{\prime}\right)-k-\left|C^{\prime}\right|-u-q=r\left(S^{\prime}, C^{\prime}, f^{\prime}\right)+n_{i}-k-u-q$. Furthermore,

$$
\begin{aligned}
d(S, C, f) & =\sum_{j=1}^{i}\left(\begin{array}{c}
S \cap X_{j} \\
2
\end{array}\right)-|C| \\
& =\sum_{j=1}^{i-1}\left(\begin{array}{c}
S^{\prime} \cap X_{j} \\
2
\end{array}\right)+\left(\begin{array}{l}
k \\
2
\end{array}\right)-\left|C^{\prime}\right|-q-u \\
& =d\left(S^{\prime}, C^{\prime}, f^{\prime}\right)+\left(\begin{array}{l}
k \\
2
\end{array}\right)-q-u
\end{aligned}
$$

We have $k=\left|S \cap X_{i}\right| \leq\left|X_{i}\right| \leq n_{i}$. We have $q \leq\left|\mathcal{P}_{2}\left(S \cap X_{i}\right)\right| \leq\left(\begin{array}{c}k \\ 2\end{array}\right)$. As $r(S, C, f)=r\left(S^{\prime}, C^{\prime}, f^{\prime}\right)+n_{i}-k-u-q$, we have $q=r^{\prime}\left(S^{\prime}, C^{\prime}, f^{\prime}\right)-r(S, C, f)+$ $n_{i}-k-u \leq r^{\prime}\left(S^{\prime}, C^{\prime}, f^{\prime}\right)$ as we have $n_{i} \leq r(S, C, f)+k+u$ as $r(S, C, f)$ is the size of the set $\bigcup_{j=1}^{i} X_{j} \backslash(S \sqcup f(C))$ and as $X_{i} \backslash\left(\left(S \cap X_{i}\right) \sqcup f\left(f^{-1}\left(X_{i}\right)\right)\right) \subseteq$ $\bigcup_{j=1}^{i} X_{j} \backslash(S \sqcup f(C))$. Thus, $q \leq r\left(S^{\prime}, C^{\prime}, f^{\prime}\right)$.

As $\left(S \cap X_{i}\right) \sqcup f\left(f^{-1}\left(X_{i}\right)\right) \subseteq X_{i}$, we deduce that $k+u \leq n_{i}$ (as $f$ is injective), thus $u \leq n_{i}-k$. As $d(S, C, f)$ is the size of the set $\bigcup_{j=1}^{i} \mathcal{P}_{2}\left(S \cap X_{j}\right) \backslash C$, we have $\mathcal{P}_{2}\left(S \cap X_{i}\right) \backslash\left(C \cap \mathcal{P}_{2}\left(S \cap X_{i}\right)\right) \subseteq \bigcup_{j=1}^{i} \mathcal{P}_{2}\left(S \cap X_{j}\right) \backslash C$. Thus, $\left(\begin{array}{c}k \\ 2\end{array}\right)-q \leq d(S, C, f)$ and so, $u=d\left(S^{\prime}, C^{\prime}, f^{\prime}\right)-d(S, C, f)+\left(\begin{array}{c}k \\ 2\end{array}\right)-q \leq d\left(S^{\prime}, C^{\prime}, f^{\prime}\right)$.

We conclude that $k, q, u$ satisfy the following inequalities:

$$
\begin{aligned}
& 0 \leq k \leq n_{i} \\
& 0 \leq q \leq \min \left(\left(\begin{array}{l}
k \\
2
\end{array}\right), r\left(S^{\prime}, C^{\prime}, f^{\prime}\right)\right) \\
& 0 \leq u \leq \min \left(n_{i}-k, d\left(S^{\prime}, C^{\prime}, f^{\prime}\right)\right)
\end{aligned}
$$

Let us show that $\left(S^{\prime}, C^{\prime}, f^{\prime}\right)$ is a nice selection of $G_{i-1}$. Suppose by contradiction that there exists a selection $\left(S^{\prime \prime}, C^{\prime \prime}, f^{\prime \prime}\right)$ of $G_{i-1}$ such that $s\left(S^{\prime \prime}, C^{\prime \prime}, f^{\prime \prime}\right)=$ $s\left(S^{\prime}, C^{\prime}, f^{\prime}\right)$ and $r\left(S^{\prime \prime}, C^{\prime \prime}, f^{\prime \prime}\right)=r\left(S^{\prime}, C^{\prime}, f^{\prime}\right)$ and that $d\left(S^{\prime \prime}, C^{\prime \prime}, f^{\prime \prime}\right)>d\left(S^{\prime}, C^{\prime}, f^{\prime}\right)$. According to Lemma 13, there exists a selection $(S p, C p, f p)$ of $G_{i}$ such that

$$
\left\{\begin{array}{l}
s(S p, C p, f p)=s\left(S^{\prime \prime}, C^{\prime \prime}, f^{\prime \prime}\right)+k \\
r(S p, C p, f p)=r\left(S^{\prime \prime}, C^{\prime \prime}, f^{\prime \prime}\right)+n_{i}-k-q-u \\
d(S p, C p, f p)=d\left(S^{\prime \prime}, C^{\prime \prime}, f^{\prime \prime}\right)+\left(\begin{array}{c}
k \\
2
\end{array}\right)-q-u
\end{array}\right.
$$

As $s\left(S^{\prime \prime}, C^{\prime \prime}, f^{\prime \prime}\right)=s\left(S^{\prime}, C^{\prime}, f^{\prime}\right), r\left(S^{\prime \prime}, C^{\prime \prime}, f^{\prime \prime}\right)=r\left(S^{\prime}, C^{\prime}, f^{\prime}\right)$ and $d\left(S^{\prime \prime}, C^{\prime \prime}, f^{\prime \prime}\right)>$ $d\left(S^{\prime}, C^{\prime}, f^{\prime}\right)$, we obtain 


$$
\begin{aligned}
& s(S p, C p, f p)=s(S, C, f) \\
& r(S p, C p, f p)=r(S, C, f) \\
& d(S p, C p, f p)>d(S, C, f)
\end{aligned}
$$

Which contradicts that $(S, C, f)$ is a nice selection of $G_{i}$. We conclude that $\left(S^{\prime}, C^{\prime}, f^{\prime}\right)$ is a nice selection of $G_{i-1}$.

We denote by $d(i, j, r)$ the maximum of $d(S, C, f)$ for any selection $(S, C, f)$ of $G_{i}$ such that $s(S, C, f)=j$ and $r(S, C, f)=r$. This quantity is set to $-\infty$ if no such selection of $G_{i}$ exists.

Lemma 15. For any integers $i, s, r$ and integers $k, u, q$ we define the following quantities:

$$
s^{\prime}=s-k, \quad r^{\prime}=r-n_{i}+u+q+k \quad \text { and } \quad d^{\prime}=d\left(i-1, s^{\prime}, r^{\prime}\right)
$$

We deduce that: $\quad d(i, s, r)=\max \left\{\left(d^{\prime}\right)+\left(\begin{array}{l}k \\ 2\end{array}\right)-q-u \mid \begin{array}{l}0 \leq k \leq n_{i} \\ 0 \leq u \leq \min \left(n_{i}-k, d^{\prime}\right) \\ \left.0 \leq q \leq \min \left(\begin{array}{l}k \\ 2\end{array}\right), r^{\prime}\right)\end{array}\right\}$

Proof. According to Lemma 13, we have

$$
d(i, s, r) \leq \max \left\{\begin{array}{l|l}
\left(d^{\prime}\right)+\left(\begin{array}{l}
k \\
2
\end{array}\right)-q-u & \begin{array}{l}
0 \leq k \leq n_{i} \\
0 \leq u \leq \min \left(n_{i}-k, d^{\prime}\right) \\
0 \leq q \leq \min \left(\left(\begin{array}{l}
k \\
2
\end{array}\right), r^{\prime}\right)
\end{array}
\end{array}\right\}
$$

as for any selection $\left(S^{\prime}, C^{\prime}, f^{\prime}\right)$ of $G_{i-1}$ such that $s\left(S^{\prime}, C^{\prime}, f^{\prime}\right)=s^{\prime}, r\left(S^{\prime}, C^{\prime}, f^{\prime}\right)=$ $r^{\prime}$ and $d\left(S^{\prime}, C^{\prime}, f^{\prime}\right)=d^{\prime}$, we can create a selection $(S, C, f)$ of $G_{i}$ such that $s(S, C, f)=s, r(S, C, f)=r$ and $d(S, C, f)=d^{\prime}+\left(\begin{array}{c}k \\ 2\end{array}\right)-q-u$.

According to Lemma 14, we have

$$
d(i, s, r) \geq \max \left\{\begin{array}{l|l}
\left(d^{\prime}\right)+\left(\begin{array}{l}
k \\
2
\end{array}\right)-q-u & \begin{array}{l}
0 \leq k \leq n_{i} \\
0 \leq u \leq \min \left(n_{i}-k, d^{\prime}\right) \\
0 \leq q \leq \min \left(\left(\begin{array}{l}
k \\
2
\end{array}\right), r^{\prime}\right)
\end{array}
\end{array}\right\}
$$

as for any selection $(S, C, f)$ of $G_{i}$ such that $s(S, C, f)=s, r(S, C, f)=r$ and $d(S, C, f)=d(i, s, r)$ we can create a nice selection $\left(S^{\prime}, C^{\prime}, f^{\prime}\right)$ of $G_{i-1}$ such that $s\left(S^{\prime}, C^{\prime}, f^{\prime}\right)=s^{\prime}, r\left(S^{\prime}, C^{\prime}, f^{\prime}\right)=r^{\prime}$ and $d\left(S^{\prime}, C^{\prime}, f^{\prime}\right)=d(S, C, f)-\left(\begin{array}{c}k \\ 2\end{array}\right)+q+u$. We deduce the equality between the two quantities.

From previous lemma we deduce the following theorem.

Theorem 4. There exists an algorithm in $O\left(n^{8}\right)$ computing the geodetic number of a complete multipartite graph with $n$ vertices.

Proof. The algorithm 2 consists mainly in six independent "for" loops. Four of them are of length at most $n$ and two of them are of length at most $n^{2}$. The complexity of the algorithm is therefore in $O\left(n^{8}\right)$. 
Notice that a complete multipartite graph can be described with the list of integers $n_{1}, \ldots, n_{k}$. In that case, the dynamic programming that we described is not polynomial if the values of the $n_{i}$ are exponential. Thus, if we formulate EdGe GeOdetic Number on complete multipartite graph as a specific problem on this class, the question whether such a problem is weak $\mathcal{N} \mathcal{P}$-hard or not is open.

\section{Conclusion}

In this paper, we investigated the hardness of the approximation of the geodetic set problems. Given our approximation lower bound for Geodetic Number and Edge Geodetic Number, the question of the existence of a $O(\log (n))$ approximation algorithm seems natural. We also proved that deciding whether a set admits a geodesic assignation NP-hard. Therefore, a second question arises: is it also hard to decide whether a set of vertices is a strong geodetic set. We also give a tight 2-approximation of this problem. Finding a lower bound for this problem is probably a good question for further work. Finally, for STRONG

Geodetic Number, we proved that it was polynomial on complete multipartite graphs. What about other graph classes?

\section{References}

1. Anand, B.S., Changat, M., Chandran, S.U.: The edge geodetic number of product graphs. In: Conference on Algorithms and Discrete Applied Mathematics. pp. 143154. Springer (2018)

2. Arokiaraj, A., Klavzar, S., Manuel, P.D., Thomas, E., Xavier, A.: Strong geodetic problems in networks. Discuss. Math. Graph Theory 40(1), 307-321 (2020)

3. Atici, M.: On the edge geodetic number of a graph. International Journal of Computer Mathematics 80(7), 853-861 (2003)

4. Berman, P., Karpinski, M.: On some tighter inapproximability results (extended abstract). In: Automata, Languages and Programming, 26th International Colloquium, ICALP'99, Prague, Czech Republic, July 11-15, 1999, Proceedings. pp. 200-209 (1999)

5. Bresar, B., Kovse, M., Tepeh, A.: Geodetic sets in graphs. In: Dehmer, M. (ed.) Structural Analysis of Complex Networks, pp. 197-218. Birkhäuser / Springer (2011)

6. Chakraborty, D., Das, S., Foucaud, F., Gahlawat, H., Lajou, D., Roy, B.: Algorithms and Complexity for Geodetic Sets on Planar and Chordal Graphs. In: Cao, Y., Cheng, S.W., Li, M. (eds.) 31st International Symposium on Algorithms and Computation (ISAAC 2020). Leibniz International Proceedings in Informatics (LIPIcs), vol. 181, pp. 7:1-7:15. Schloss Dagstuhl-Leibniz-Zentrum für Informatik (2020)

7. Chakraborty, D., Foucaud, F., Gahlawat, H., Ghosh, S.K., Roy, B.: Hardness and approximation for the geodetic set problem in some graph classes. In: Changat, M., Das, S. (eds.) Algorithms and Discrete Applied Mathematics - 6th International Conference, CALDAM 2020, Hyderabad, India, February 13-15, 2020, Proceedings. Lecture Notes in Computer Science, vol. 12016, pp. 102-115. Springer (2020)

8. Darmann, A., Döcker, J.: On simplified NP-complete variants of monotone 3-sat. Discret. Appl. Math. 292, 45-58 (2021) 
9. Dinur, I., Steurer, D.: Analytical approach to parallel repetition. In: Proceedings of the Forty-Sixth Annual ACM Symposium on Theory of Computing. p. 624-633. STOC '14, Association for Computing Machinery, New York, NY, USA (2014)

10. Doughat, A., Kong, M.: Computing geodetic bases of chordal and split graphs. Journal Of Combinatorial Mathematics And Combinatorial Computing 22, 67-78 (1996)

11. Douthat, A., Kong, M.: Computing the geodetic number of bipartite graphs. Congressus Numerantium pp. 113-120 (1995)

12. Gledel, V., Irsic, V., Klavzar, S.: Strong geodetic cores and cartesian product graphs. Appl. Math. Comput. 363 (2019)

13. Harary, F., Loukakis, E., Tsouros, C.: The geodetic number of a graph. Mathematical and Computer Modelling 17(11), 89-95 (1993)

14. Iršič, V.: Strong geodetic number of complete bipartite graphs and of graphs with specified diameter. Graphs Comb. 34(3), 443-456 (2018)

15. Iršič, V., Konvalinka, M.: Strong geodetic problem on complete multipartite graphs. Ars Math. Contemp. 17(2), 481-491 (2019)

16. Klavzar, S., Manuel, P.D.: Strong geodetic problem in grid-like architectures. Bulletin of the Malaysian Mathematical Sciences Society 41(3), 1671-1680 (2018)

17. Manuel, P., Klavžar, S., Xavier, A., Arokiaraj, A., Thomas, E.: Strong edge geodetic problem in networks. Open Mathematics 15(1), 1225-1235 (2017)

18. Mezzini, M.: An o (mn2) algorithm for computing the strong geodetic number in outerplanar graphs. Discuss. Math. Graph Theory (2020)

19. Papadimitriou, C.H., Yannakakis, M.: Optimization, approximation, and complexity classes 43(3), 425-440 (1991)

20. Rehmani, S., Sunitha, M.: Edge geodesic number of a fuzzy graph. Journal of Intelligent \& Fuzzy Systems 37(3), 4273-4286 (2019)

21. Santhakumaran, A., Ullas Chandran, S.: The edge geodetic number and cartesian product of graphs. Discussiones Mathematicae Graph Theory 30(1), 55-73 (2010)

22. Santhakumaran, A., John, J.: Edge geodetic number of a graph. Journal of Discrete Mathematical Sciences and Cryptography 10(3), 415-432 (2007)

23. Santhakumaran, A., Ullas Chandran, S.: Comment on" edge geodetic covers in graphs. Proyecciones (Antofagasta) 34(4), 343-350 (2015)

24. Weller, M., Chateau, A., Giroudeau, R., König, J., Pollet, V.: On residual approximation in solution extension problems. J. Comb. Optim. 36(4), 1195-1220 (2018)

25. Zmazek, E.: Strong edge geodetic problem on grids. Bulletin of the Malaysian Mathematical Sciences Society pp. 1-20 (2021) 


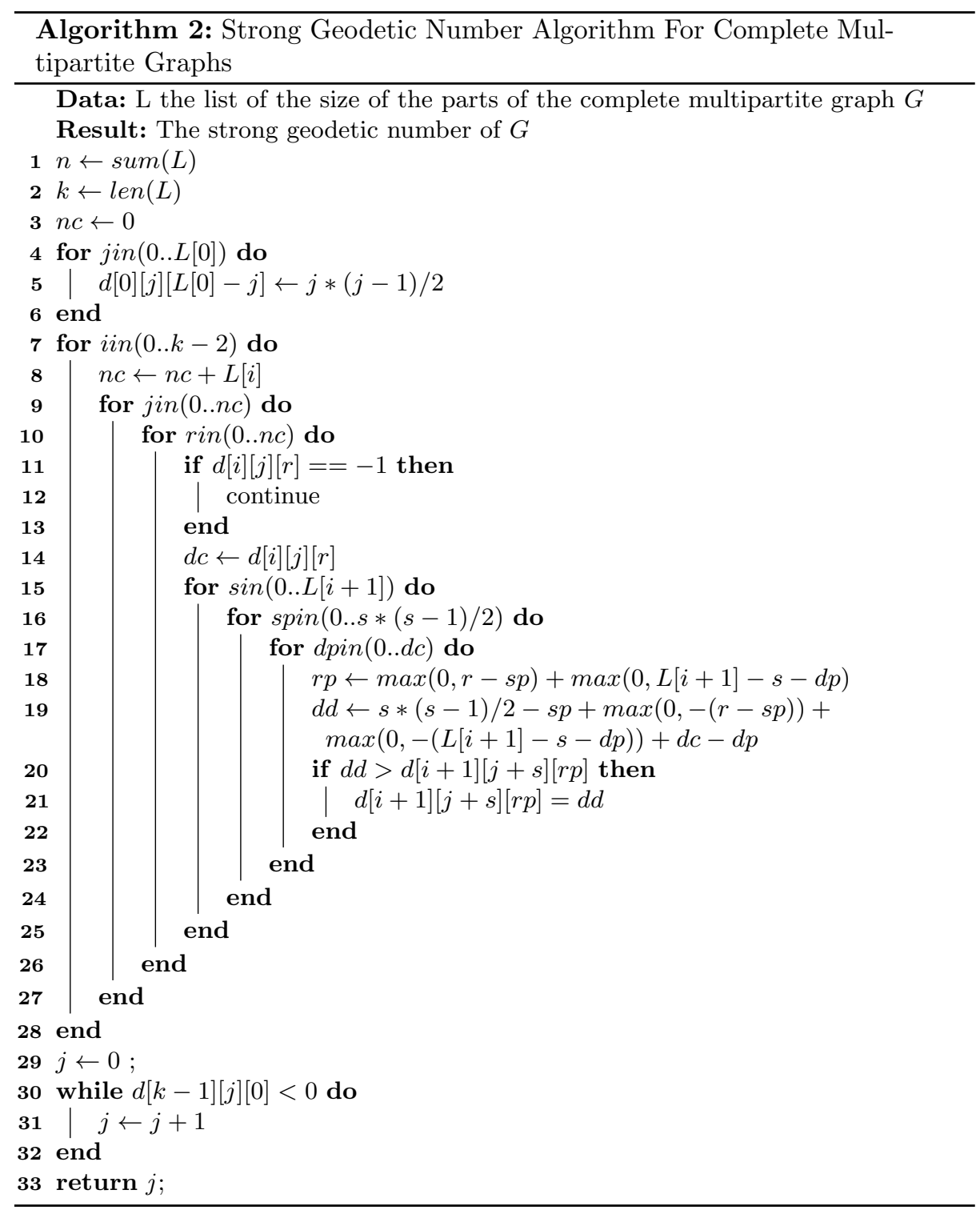

